\title{
The energy density of martensitic thin films via dimension reduction
}

\author{
LORENZO FREDDI ${ }^{\dagger}$ \\ Dipartimento di Matematica e Informatica, Università di Udine, \\ via delle Scienze 206, 33100 Udine, Italy \\ AND \\ ROBERTO PARONI \\ Dipartimento di Architettura e Pianificazione, Università di Sassari, \\ Palazzo del Pou Salit, Piazza Duomo, 07041 Alghero, Italy
}

[Received 27 November 2003 and in revised form 29 April 2004]

\begin{abstract}
A variational limit defined on the space of bi-dimensional gradient Young measures is obtained from three-dimensional elasticity via dimension reduction. The resulting limit problem uniquely determines the energy density of the thin film. Our result might be used to compute the microstructure in membranes made of phase transforming material.
\end{abstract}

\section{Introduction}

Martensitic thin films have recently attracted much interest because of their applications in the construction of actuators, sensors and micromachines. These technological applications have generated a new interest in nonlinear membranes theories. The purpose of the present paper is to obtain, by means of a rigorous dimension reduction, the energy density of a thin film, and hence to deduce a membrane model capable of describing the microstructure observed in martensitic materials.

Phase transformations have been successfully modelled within the theory of non-linear elasticity by Ball and James [5]. Within this approach, equilibrium configurations are found by minimizing the total free energy of the body.

In the spirit of the works on dimension reduction (see for instance [1, 3]) we start by considering the total free energy

$$
I_{\varepsilon}(y)=\int_{\Omega_{\varepsilon}} W(D y) \mathrm{d} x-\int_{\Omega_{\varepsilon}} \hat{f}^{\varepsilon} \cdot y \mathrm{~d} x
$$

corresponding to a deformation $y$ of a hyperelastic three-dimensional body which occupies the cylindrical region $\Omega_{\varepsilon}:=\omega \times(-\varepsilon / 2, \varepsilon / 2)$, with Helmholtz free energy $W$ and subject to some body forces $\hat{f}^{\varepsilon}$ and boundary conditions.

Under the usual assumptions on $W$ and $\hat{f}^{\varepsilon}$ there are different topologies which ensure compactness of the family of minimizers (or quasi-minimizers). Once one of these topologies is chosen, the membrane model is obtained by passing to the limit as $\varepsilon \rightarrow 0$ in an appropriate variational sense ( $\Gamma$-convergence). Roughly speaking, this variational limit ensures the convergence, in such

\footnotetext{
${ }^{\dagger}$ Email: freddi@dimi.uniud.it

Email: paroni@uniss.it
} 
topology, of minimizers of the energy at level $\varepsilon$ to the minimizers of the membrane problem. The resulting limit problem depends on the chosen topology.

Le Dret and Raoult [22], working with the strong convergence in $L^{p}$, for an appropriate $p$ larger than 1 related to the growth of $W$, have found a limit energy of the form

$$
J(y)=\int_{\omega} Q W_{0}\left(D_{\alpha} y\right) \mathrm{d} x_{\alpha}-\int_{\omega} \mathrm{m}_{3} f \cdot y \mathrm{~d} x_{\alpha},
$$

where $Q W_{0}$ denotes the quasiconvex envelope of the function

$$
W_{0}(\bar{F}):=\min \left\{W(\bar{F} \mid z): z \in \mathbb{R}^{3}\right\}, \quad \bar{F} \in M^{3 \times 2} .
$$

By $D_{\alpha} y=\left(D_{1} y, D_{2} y\right)$ we mean the gradient of the admissible deformation $y=y\left(x_{\alpha}\right)$, $x_{\alpha}=\left(x_{1}, x_{2}\right)$. At this introductory level it is useless to specify the meaning $\operatorname{of}_{3} f$, for which we refer to Section 4. A similar result in the case of strings was previously obtained by Acerbi, Buttazzo and Percivale in [1]. Typically, the infimum of the total free energy $I_{\varepsilon}$ of a martensitic material is not attained. In general, the minimizing sequences develop fine scale oscillations, which, according to the interpretation due to Ball and James [5, 6], model the microstructure experimentally observed in specimens of phase transforming materials. On the other hand the minimum of the functional $J$ is achieved but the limit problem, when applied to martensitic materials, does not describe the microstructure. Our aim is to derive a thin film theory capable of capturing the oscillations of the minimizing sequences. Inspired by earlier works on phase transitions by Ball and James [6], James and Kinderlehrer [19], Kinderlehrer and Pedregal [20, 21], we take the variational limit in the framework of parametrized measures introduced by L. C. Young [31], which is one of the most successful tools used to characterize the oscillatory behaviour of sequences of functions. To this end we extend the three-dimensional energy functionals to the space of Young measures associating to each gradient $D y$ the corresponding Young measure $\mu_{x}=\delta_{D y(x)}$; a similar idea was used by Paroni [26]. Since we are interested in a bi-dimensional limit, we consider the average over the thickness of the cylinders of these measures $\mu_{x}$. Then, under the weak* convergence of the averaged gradient Young measures, we obtain the limit energy

$$
I(v)=\int_{\omega} \int_{M^{3 \times 2}} W_{0}(\bar{F}) \mathrm{d} v_{x_{\alpha}}(\bar{F}) \mathrm{d} x_{\alpha}-\int_{\omega} \mathrm{m}_{3} f \cdot y \mathrm{~d} x_{\alpha},
$$

whose domain is the space of bi-dimensional gradient Young measures satisfying the appropriate boundary conditions. The functionals $I$ and $J$ share the same infimum value, but there is a major difference between them: while $J$ is uncapable of capturing the oscillations of the minimizing sequences, the functional $I$ characterizes the microstructure through the Young measure solution. Indeed, the minimizers of $I$ carry more information than those of $J$ since the minimizers of $I$ provide a description of the microstructure and their centers of mass select minimizers of $J$ (see Section 6). The computation of the microstructure in a thin film can therefore be evaluated through the use of the functional $I$ exactly as is done in three-dimensional elasticity (see for instance Ball and James [5, 6], and Luskin [23]).

Another important feature of the limit functional $I$, which is missing in all the other previously obtained variational problems, and which is also relevant from the point of view of the computation of the microstructure, is that it uniquely determines the energy density $W_{0}$ of the thin film (see Section 7). The total energy of the membrane, written without using Young measures, is therefore

$$
E(y)=\int_{\omega} W_{0}\left(D_{\alpha} y\right) \mathrm{d} x_{\alpha}-\int_{\omega} \mathrm{m}_{3} f \cdot y \mathrm{~d} x_{\alpha} .
$$


Thin films of martensitic material have also been studied by Bhattacharya and James [10]. These authors have considered a body characterized not only by a free energy but also by an interfacial energy; indeed they have taken a total energy of the form

$$
I_{\varepsilon, \kappa}(y)=\int_{\Omega_{\varepsilon}}\left(W(D y)+\kappa\left|D^{2} y\right|^{2}\right) \mathrm{d} x-\int_{\Omega_{\varepsilon}} \hat{f}^{\varepsilon} \cdot y \mathrm{~d} x,
$$

where $\kappa$ is a positive constant, $W$ has a quadratic growth and $D^{2} y$ denotes the Hessian of $y$. The limit problem

$$
J_{\kappa}(y, b)=\int_{\omega}\left(W\left(D_{\alpha} y \mid b\right)+\kappa\left(\left|D_{\alpha}^{2} y\right|^{2}+2\left|D_{\alpha} b\right|^{2}\right)\right) \mathrm{d} x_{\alpha}-\int_{\omega} \mathrm{m}_{3} f \cdot y \mathrm{~d} x_{\alpha}
$$

was obtained by them under the weak convergence in $W^{2,2}$ of the deformation to $y$ and the weak convergence in $W^{1,2}$ to the Cosserat vector $b$ of the third partial derivative of a suitable rescaling of the deformation. We note that the interfacial energy plays a crucial role in order to supply a limit problem in which no quasiconvexification appears. On the other hand, it has been shown by Shu [29] that if we also let $\kappa \rightarrow 0$ the variational limit coincides with that obtained by Le Dret and Raoult.

Bělík and Luskin [7, 8] have observed that the deformations with finite energy cannot have sharp interfaces between compatible variants, unless the constant $\kappa$ is set equal to 0 . For this reason they set the problem in the framework of functions with bounded Hessian and considered an interfacial energy proportional to the total variation of the deformation gradient.

More recently Bocea [11] has studied the limit $\mathcal{W}(y, b)$ of $I_{\varepsilon}$ under the weak convergence in $W^{1, p}$ of the deformations to $y$ and the weak convergence in $L^{p}$ to $b$ of the third partial derivative of a suitable rescaling of the deformation. Using a result of Bocea and Fonseca [12], he has proved that

$$
\mathcal{W}(y, b)=\inf \int_{\Omega \times M^{3 \times 3}} W \mathrm{~d} \mu
$$

where the infimum is taken over all Young measures $\mu$ generated by sequences of gradients of deformations whose third partial derivative is suitably rescaled.

We conclude this brief review by mentioning also the papers [9, 13, 28], among the wide literature on thin films, and a related paper by the authors [18] where some of the ideas used in this paper are applied to obtain the energy density of a martensitic string starting from a three-dimensional body with a free energy that becomes infinite when the volume locally vanishes.

The paper is organized as follows. In Sections 2 and 3 we summarize well known definitions and properties of Young measures and $\Gamma$-convergence. In Section 4 we consider the three-dimensional problems and set the framework in which the $\Gamma$-limit will be taken. Moreover we briefly recall the earlier result of Le Dret and Raoult. In Section 5 various preliminary results are proved and the main coerciveness and $\Gamma$-convergence theorems are stated. The proofs are postponed to Section 8 . In Section 6 we study the relationship between our limit functional and the one obtained by Le Dret and Raoult. The energy density of the thin film is proposed in Section 7.

\section{Young measures preliminaries}

The main references for this section are Ball [4], Müller [24] and Pedregal [27].

Throughout the whole section $\Omega$ is an open bounded subset of $\mathbb{R}^{k}$. We denote by $M^{h \times k}$ the set of $h \times k$ real matrices which will be often identified with the Euclidean space $\mathbb{R}^{h k}$. By $\mathcal{M}\left(M^{h \times k}\right)$ 
we mean the space of $\mathbb{R}$-valued Borel measures on $M^{h \times k}$ which can be viewed as the dual of the separable Banach space $C_{0}\left(M^{h \times k}\right)$ under the duality

$$
\langle\mu, \varphi\rangle=\int_{M^{h \times k}} \varphi \mathrm{d} \mu .
$$

We recall that a mapping $\mu: \Omega \rightarrow \mathcal{M}\left(M^{h \times k}\right)$ is said to be weak ${ }^{*}$ measurable whenever the function $x \mapsto\langle\mu(x), \varphi\rangle$ is measurable for every $\varphi \in C_{0}\left(M^{h \times k}\right)$. The space $L_{w}^{\infty}\left(\Omega ; \mathcal{M}\left(M^{h \times k}\right)\right)$ consists of all weak* measurable mappings $\mu: \Omega \rightarrow \mathcal{M}\left(M^{h \times k}\right)$ which are essentially bounded. This space will be endowed with the weak* topology induced by the duality with $L^{1}\left(\Omega ; C_{0}\left(M^{h \times k}\right)\right)$. Therefore, a sequence $\mu^{n}$ is weak ${ }^{*}$ converging to a limit $\mu$ if and only if

$$
\int_{\Omega}\left\langle\mu_{x}^{n}, \varphi\right\rangle g(x) \mathrm{d} x \rightarrow \int_{\Omega}\left\langle\mu_{x}, \varphi\right\rangle g(x) \mathrm{d} x, \quad \forall \varphi \in C_{0}\left(M^{h \times k}\right), \forall g \in L^{1}(\Omega) .
$$

A Young measure on $\Omega$ with target space $M^{h \times k}$ is an element $v$ of the space $L_{w}^{\infty}\left(\Omega ; \mathcal{M}\left(M^{h \times k}\right)\right)$ such that $v_{x}:=v(x)$ is a probability measure for almost every $x \in \Omega$.

A Young measure $\mu \in L_{w}^{\infty}\left(\Omega ; \mathcal{M}\left(M^{h \times k}\right)\right)$ is said to be generated by the sequence of measurable functions $u^{n}: \Omega \rightarrow M^{h \times k}$ if

$$
\delta_{u^{n}(\cdot)} \rightarrow \mu \quad \text { weak }^{*} \text { in } L_{w}^{\infty}\left(\Omega ; \mathcal{M}\left(M^{h \times k}\right)\right) .
$$

Every Young measure is generated by some sequence of measurable functions.

The center of mass of a Young measure $\mu \in L_{w}^{\infty}\left(\Omega ; \mathcal{M}\left(M^{h \times k}\right)\right)$ is the function

$$
x \mapsto\left\langle\mu_{x}, \mathrm{id}\right\rangle=\int_{M^{h \times k}} \lambda \mathrm{d} \mu_{x}(\lambda),
$$

where id denotes the identity mapping.

In this paper an important role will be played by the image of a measure and by the fiber product of two Young measures. Let $(X, \mathcal{A}, \mu)$ and $(Y, \mathcal{B})$ be measurable spaces and $\theta: X \rightarrow Y$. Then the image of the measure $\mu$ under the mapping $\theta$ is defined by $\theta_{\#} \mu(A):=\mu\left(\theta^{-1}(A)\right)$ for every $A \in \mathcal{B}$.

The fiber product of two Young measures $v \in L_{w}^{\infty}\left(\Omega ; \mathcal{M}\left(\mathbb{R}^{m}\right)\right)$ and $\sigma \in L_{w}^{\infty}\left(\Omega ; \mathcal{M}\left(\mathbb{R}^{s}\right)\right)$ is the Young measure $\mu \in L_{w}^{\infty}\left(\Omega ; \mathcal{M}\left(M^{m \times s}\right)\right)$ usually denoted by $\mu=v \otimes \sigma$ and defined by

$$
\mu_{x}=v_{x} \otimes \sigma_{x}
$$

where $\otimes$ denotes the usual tensor product of measures. The following theorem of Balder and Valadier (see Valadier [30, Theorem 14]) gives a sufficient condition which allows one to pass to the limit in the fiber product.

THEOREM 2.1 Let $v^{n}$ and $\sigma^{n}$ be two sequences of Young measures belonging to $L_{w}^{\infty}\left(\Omega ; \mathcal{M}\left(\mathbb{R}^{m}\right)\right)$ and $L_{w}^{\infty}\left(\Omega ; \mathcal{M}\left(\mathbb{R}^{s}\right)\right)$ respectively, such that

$$
v^{n} \rightarrow v \quad \text { weak }^{*} \text { in } L_{w}^{\infty}\left(\Omega ; \mathcal{M}\left(\mathbb{R}^{m}\right)\right)
$$

while

$$
\sigma^{n} \rightarrow \sigma=\delta_{u(\cdot)} \quad \text { weak }^{*} \text { in } L_{w}^{\infty}\left(\Omega ; \mathcal{M}\left(\mathbb{R}^{s}\right)\right)
$$

for a suitable measurable function $u$. Then

$$
v^{n} \otimes \sigma^{n} \rightarrow v \otimes \sigma \quad \text { weak }^{*} \text { in } L_{w}^{\infty}\left(\Omega ; \mathcal{M}\left(\mathbb{R}^{m+s}\right)\right) .
$$


Extensive use will be made of the following theorem (see for instance Müller [24, Corollary 3.3]) which states the fundamental property of Young measures.

THEOREM 2.2 Suppose that the sequence of maps $u^{n}: \Omega \rightarrow \mathbb{R}^{h}$ generates the Young measure $v$. Let $f: \Omega \times \mathbb{R}^{h} \rightarrow \mathbb{R}$ be a Carathéodory function and assume that the negative part $f^{-}\left(x, u^{n}(x)\right)$ is weakly relatively compact in $L^{1}(\Omega)$. Then

$$
\liminf _{k \rightarrow \infty} \int_{\Omega} f\left(x, u^{n}(x)\right) \mathrm{d} x \geqslant \int_{\Omega} \int_{\mathbb{R}^{h}} f(x, \lambda) \mathrm{d} v_{x}(\lambda) \mathrm{d} x .
$$

If, in addition, the sequence of functions $x \mapsto|f|\left(x, u^{n}(x)\right)$ is weakly relatively compact in $L^{1}(\Omega)$ then

$$
f\left(\cdot, u^{n}(\cdot)\right) \rightarrow \bar{f} \quad \text { weakly in } L^{1}(\Omega) \text { where } \bar{f}(x)=\int_{\mathbb{R}^{h}} f(x, \lambda) \mathrm{d} v_{x}(\lambda) .
$$

We are mainly interested in those Young measures which are generated by sequences of gradients, in the sense specified by the following definition.

Definition 2.3 A weak* measurable map $v: \Omega \rightarrow \mathcal{M}\left(M^{h \times k}\right)$ is a $W^{1, p}$-gradient Young measure if there exists a sequence of maps $u^{n}: \Omega \rightarrow \mathbb{R}^{h}$ such that $u^{n} \rightarrow u$ weakly in $W^{1, p}\left(\Omega ; \mathbb{R}^{h}\right)$ and $\delta_{D u^{n}(\cdot)} \rightarrow v$ weak* in $L_{w}^{\infty}\left(\Omega ; M^{h \times k}\right)$. In this case, the $W^{1, p}$-gradient Young measure $v$ is said to be generated by the sequence $D u^{n}$ and $u$ is called an underlying deformation for $v$.

Gradient Young measures are in a sort of duality with quasiconvex functions.

DEFinition 2.4 A function $f: M^{h \times k} \rightarrow(-\infty,+\infty]$ is said to be quasiconvex if for every open and bounded subset $A$ of $\mathbb{R}^{k}$ with negligible boundary (with respect to the Lebesgue measure) one has

$$
\int_{A} f(F+D \varphi) \mathrm{d} x \geqslant \int_{A} f(F) \mathrm{d} x=|A| f(F) \quad \forall \varphi \in C_{0}^{1}\left(A ; \mathbb{R}^{h}\right), \forall F \in M^{h \times k},
$$

whenever the integral on the left hand side exists $(|A|$ denotes the Lebesgue measure of the open set $A$ ).

The following characterization of $W^{1, p}$-gradient Young measures is due to Kinderlehrer and Pedregal [21].

THEOREM 2.5 Let $p \in[1,+\infty)$. A weak* measurable map $v: \Omega \rightarrow \mathcal{M}\left(M^{h \times k}\right)$ is a $W^{1, p}$-gradient Young measure if and only if $v_{x}$ is a probability measure a.e. and the following three conditions hold:

(i) $\int_{\Omega} \int_{M^{h \times k}}|F|^{p} \mathrm{~d} v_{x}(F) \mathrm{d} x<+\infty$;

(ii) $\left\langle v_{x}\right.$, id $\rangle=D u(x), u \in W^{1, p}\left(\Omega ; \mathbb{R}^{h}\right)$;

(iii) $\left\langle v_{x}, f\right\rangle \geqslant f\left(\left\langle v_{x}\right.\right.$, id $\left.\rangle\right)$ for almost every $x$ and all quasiconvex $f$ with $|f|(F) \leqslant C\left(|F|^{p}+1\right)$.

The following proposition (see for instance Pedregal [27, Lemma 8.3]), sometimes called Zhang's Lemma, is a variant of a result obtained by Acerbi and Fusco [2].

Proposition 2.6 Let $p \in[1,+\infty]$ and let $v$ be a $W^{1, p}$-gradient Young measure. If $u_{0} \in$ $W^{1, p}\left(\Omega ; \mathbb{R}^{h}\right)$ is an underlying deformation for $v$ then there exists a sequence $u^{n} \in C_{0}^{\infty}\left(\Omega ; \mathbb{R}^{h}\right)$ such that $u^{n} \rightarrow 0$ weakly in $W_{0}^{1, p}\left(\Omega ; \mathbb{R}^{h}\right),\left|D u^{n}\right|^{p}$ are equi-integrable $\left(\left|D u^{n}\right|\right.$ equi-bounded in the case $p=+\infty)$ and $D\left(u_{0}+u^{n}\right)$ generates $v$. 


\section{Sequential $\Gamma$-convergence}

For the purposes of this paper we need a kind of variational convergence which allows us to treat families of functionals $F_{\varepsilon}: X \rightarrow \overline{\mathbb{R}}=(-\infty,+\infty]$ defined on a space which may be different from the domain of the limiting functional. It is a variant of De Giorgi's $\Gamma$-convergence, and has been introduced by Anzellotti, Baldo and Percivale in [3] in order to study dimension reduction problems in mechanics. Let $X$ be a set, let $(Y, \tau)$ be a topological space and let $q: X \rightarrow Y$. Given a sequence of functionals $F_{n}: X \rightarrow \overline{\mathbb{R}}$ and a point $y \in Y$, define

$$
\begin{aligned}
\Gamma(q, \tau Y) \liminf _{n \rightarrow \infty} F_{n}(y) & :=\inf \left\{\liminf _{n \rightarrow \infty} F_{n}\left(x_{n}\right): q\left(x_{n}\right) \stackrel{\tau}{\rightarrow} y\right\}, \\
\Gamma(q, \tau Y) \limsup _{n \rightarrow \infty} F_{n}(y) & :=\inf \left\{\limsup _{n \rightarrow \infty} F_{n}\left(x_{n}\right): q\left(x_{n}\right) \stackrel{\tau}{\rightarrow} y\right\} ;
\end{aligned}
$$

these are called, respectively, the sequential $\Gamma^{-}$-lower and $\Gamma^{-}$-upper limit at the point $y$. In 3.1, as in what follows, we have dropped the minus sign for notational simplicity.

Definition 3.1 Given a sequence $\varepsilon_{n}$ of positive numbers we say that a sequence $F_{\varepsilon_{n}}: X \rightarrow \overline{\mathbb{R}}$ (sequentially) $\Gamma(q, \tau Y)$-converges to a functional $F: Y \rightarrow \overline{\mathbb{R}}$ at a point $y \in Y$, and we write

$$
\Gamma(q, \tau Y) \lim _{n \rightarrow \infty} F_{\varepsilon_{n}}(y)=F(y),
$$

if

$$
\Gamma(q, \tau Y) \liminf _{n \rightarrow \infty} F_{\varepsilon_{n}}(y)=\Gamma(q, \tau Y) \limsup _{n \rightarrow \infty} F_{\varepsilon_{n}}(y)=F(y) .
$$

We say that a family of functionals $F_{\varepsilon}: X \rightarrow \overline{\mathbb{R}}, \varepsilon>0$, (sequentially) $\Gamma(q, \tau Y)$-converges to a functional $F: Y \rightarrow \overline{\mathbb{R}}$ at a point $y \in Y$, and we write

$$
\Gamma(q, \tau Y) \lim _{\varepsilon \rightarrow 0} F_{\varepsilon}(y)=F(y),
$$

if for any sequence $\varepsilon_{n}$ of positive reals such that $\lim _{n \rightarrow \infty} \varepsilon_{n}=0$ we have

$$
\Gamma(q, \tau Y) \lim _{n \rightarrow \infty} F_{\varepsilon_{n}}(y)=F(y) .
$$

We say that a family of functionals $\Gamma(q, \tau Y)$-converges on a set if it $\Gamma(q, \tau Y)$-converges at every point of the set.

REMARK 3.2 Let us remark that 3.2 holds if and only if the following two conditions are satisfied:

1. for every sequence $x_{n} \in X$ such that $q\left(x_{n}\right) \stackrel{\tau}{\rightarrow} y$ one has

$$
\liminf _{n \rightarrow \infty} F_{\varepsilon_{n}}\left(x_{n}\right) \geqslant F(y)
$$

2. there exists a sequence $\bar{x}_{n} \in X$ such that $q\left(\bar{x}_{n}\right) \stackrel{\tau}{\rightarrow} y$ and

$$
\lim _{n \rightarrow \infty} F_{\varepsilon_{n}}\left(\bar{x}_{n}\right)=F(y) .
$$


If $X=Y$ and $q$ is the identity map, then the $\Gamma$-limits defined above are the classical De Giorgi sequential $\Gamma^{-}$-limits which will be denoted by the classical notation

$$
\Gamma^{-}(\tau X) \lim _{\varepsilon \rightarrow 0} F_{\varepsilon}=F .
$$

Moreover, it is easy to see that if we set

$$
I_{\varepsilon}(y)=\inf \left\{F_{\varepsilon}(x): q(x)=y\right\}, \quad \varepsilon>0,
$$

then (3.3) holds true if and only if

$$
\Gamma^{-}(\tau Y) \lim _{\varepsilon \rightarrow 0} I_{\varepsilon}(y)=F(y) .
$$

Besides the notion of sequential $\Gamma$-convergence, also a more general concept of $\Gamma$-convergence, associated with a topology on $X$, can be introduced (see for instance Dal Maso [17]), and the two kinds of $\Gamma$-convergence coincide on first countable topological spaces. Hence when the topological space $(Y, \tau)$ is first countable and the family $F_{\varepsilon}$ is equi-coercive, then the $\Gamma(q, \tau Y)$-convergence has a variational character which is typical of $\Gamma$-convergence, that is, it ensures the lower semicontinuity of the limit, which, moreover, turns out to be coercive. Roughly speaking, it preserves convergence of minima and of minimizers. This is summarized by the statements below.

Definition 3.3 The family $F_{\varepsilon}$ is said to be $(q, \tau Y)$-equi-coercive if for every real number $M$ there exists a $\tau$-compact and $\tau$-closed subset $K_{M}$ of $Y$ such that

$$
\left\{q(x): F_{\varepsilon}(x) \leqslant M\right\} \subseteq K_{M} \quad \text { for every } \varepsilon>0 .
$$

This definition reduces to the classical one of $\tau$-equi-coerciveness in the case $q=\mathrm{id}$.

Proposition 3.4 Assume that $\Gamma(q, \tau Y) \lim _{\varepsilon \rightarrow 0} F_{\varepsilon}=F$ on $Y$ and that the family $F_{\varepsilon}$ is $(q, \tau Y)$ equi-coercive. Then:

(i) $F$ is $\tau$-lower semicontinuous;

(ii) $F$ is $\tau$-coercive;

(iii) if $x_{\varepsilon} \in X$ satisfy $\liminf _{\varepsilon \rightarrow 0} F_{\varepsilon}\left(x_{\varepsilon}\right)=\liminf _{\varepsilon \rightarrow 0} \inf F_{\varepsilon}$ (e.g. if $x_{\varepsilon}$ minimizes $F_{\varepsilon}$ ) then

(a) if $\varepsilon_{n}$ is a sequence such that $\lim _{n \rightarrow \infty} \varepsilon_{n}=0$ and if $q\left(x_{\varepsilon_{n}}\right) \stackrel{\tau}{\rightarrow} y$ then $y$ is a minimizer of $F$ on $Y$ and $\lim _{n \rightarrow \infty} F_{\varepsilon_{n}}\left(x_{\varepsilon_{n}}\right)=F(y)$;

(b) there is a sequence $\varepsilon_{n}$ such that $\lim _{n \rightarrow \infty} \varepsilon_{n}=0$ and a minimizer $y$ of $F$ on $Y$ such that $q\left(x_{\varepsilon_{n}}\right) \stackrel{\tau}{\rightarrow} y$.

Proposition 3.5 If $Y$ is the dual of a separable Banach space, $\tau$ is the weak* topology, and $F_{n}: X \rightarrow \overline{\mathbb{R}}$ is $(q, \tau Y)$-equi-coercive, then

$$
\Gamma(q, \tau Y) \lim _{n \rightarrow \infty} F_{n}(y)=F(y)
$$

if and only if

(i) $\liminf _{n \rightarrow \infty} F_{n}\left(x_{n}\right) \geqslant F(y)$ for every sequence $x_{n} \in X$ such that $q\left(x_{n}\right) \stackrel{\tau}{\rightarrow} y$;

(ii) for every sequence $\left(n_{k}\right)$ of positive integers there is a subsequence $\left(n_{k_{p}}\right)$ and a sequence $x_{p} \in X$ such that

$$
q\left(x_{p}\right) \stackrel{\tau}{\rightarrow} y \text { and } \quad \lim _{p \rightarrow \infty} F_{n_{k_{p}}}\left(x_{p}\right)=F(y)
$$


Proof. This is an easy consequence of the weak* metrizability of compact subsets of $Y$ and of the Urysohn property of $\Gamma$-convergence (see for instance Dal Maso [17, Chapter 8]).

\section{Setting of the problem and previous results}

For all $\varepsilon>0$, let $\Omega_{\varepsilon}=\left\{x=\left(x_{\alpha}, x_{3}\right) \in \mathbb{R}^{2} \times \mathbb{R}: x_{\alpha} \in \omega,\left|x_{3}\right|<\varepsilon / 2\right\}$, where $\omega$ is an open, bounded subset of $\mathbb{R}^{2}$ with Lipschitz boundary. We can think of $\Omega_{\varepsilon}$ as the reference configuration of a hyperelastic homogeneous body with stored energy density function $W: M^{3 \times 3} \rightarrow \mathbb{R}$. This function $W$ is assumed to be continuous and to satisfy the growth assumption

$$
c\left(|F|^{p}-1\right) \leqslant W(F) \leqslant C\left(|F|^{p}+1\right)
$$

for two constants $C \geqslant c>0$. The total energy $I_{\varepsilon}$ of the body is given by

$$
I_{\varepsilon}(y)=\int_{\Omega_{\varepsilon}} W(D y) \mathrm{d} x-\int_{\Omega_{\varepsilon}} \hat{f}^{\varepsilon} \cdot y \mathrm{~d} x,
$$

where the body force density $\hat{f}^{\varepsilon}$ is taken in $L^{q}\left(\Omega_{\varepsilon} ; \mathbb{R}^{3}\right)$, with $1 / p+1 / q=1$. Assuming, for the sake of simplicity, the body to be clamped on the lateral surface $\Gamma_{\varepsilon}:=\partial \omega \times(-\varepsilon / 2, \varepsilon / 2)$ of $\Omega_{\varepsilon}$, the equilibrium configuration will be found by minimizing the energy $I_{\varepsilon}$ over the set of admissible deformations

$$
\mathcal{A}_{\varepsilon}=\left\{y \in W^{1, p}\left(\Omega_{\varepsilon} ; \mathbb{R}^{3}\right): y(x)=x \text { on } \Gamma_{\varepsilon}\right\} .
$$

In order to study the behaviour of the minimizers $y^{\varepsilon}$ of $I_{\varepsilon}$ as $\varepsilon \rightarrow 0$, it is convenient to transform, after Ciarlet and Destuynder [15], the problems under consideration into problems over a fixed domain. To this end we define $\Omega:=\Omega_{1}, \Gamma:=\Gamma_{1}$ and a map $\theta_{\varepsilon}: L^{p}\left(\Omega_{\varepsilon} ; \mathbb{R}^{3}\right) \rightarrow L^{p}\left(\Omega ; \mathbb{R}^{3}\right)$ by

$$
\left(\theta_{\varepsilon} y\right)(x)=y\left(x_{1}, x_{2}, \varepsilon x_{3}\right) .
$$

We accordingly rescale the energies by setting $I_{\varepsilon}^{\Omega}(y)=I_{\varepsilon}\left(\theta_{\varepsilon}^{-1} y\right) / \varepsilon$, which is

$$
I_{\varepsilon}^{\Omega}(y):=\int_{\Omega} W\left(D_{\alpha} y \mid \frac{D_{3} y}{\varepsilon}\right) \mathrm{d} x-\int_{\Omega} f^{\varepsilon} \cdot y \mathrm{~d} x,
$$

where we have set $f^{\varepsilon}:=\theta_{\varepsilon} \hat{f}^{\varepsilon}$. Following Le Dret and Raoult [22] we assume that $f^{\varepsilon}$ does not depend on $\varepsilon$ and set $f:=f^{\varepsilon}$. The admissible set over which the total energy has to be minimized becomes

$$
\mathcal{A}_{\varepsilon}^{\Omega}=\left\{y \in W^{1, p}\left(\Omega ; \mathbb{R}^{3}\right): y(x)=\left(x_{1}, x_{2}, \varepsilon x_{3}\right) \text { on } \Gamma\right\} .
$$

Above and in what follows, we identify $M^{3 \times 3}$ with $M^{3 \times 2} \times \mathbb{R}^{3}$, and write $(\bar{F} \mid z)$ for the element in $M^{3 \times 3}$ which is identified with $(\bar{F}, z) \in M^{3 \times 2} \times \mathbb{R}^{3}$. Moreover, $D_{\alpha} y$ denotes the first two columns of the gradient of $y$.

Within a similar framework, Le Dret and Raoult ([22, Theorem 2]) have proved that, as $\varepsilon \rightarrow 0$, the family of functionals

$$
J_{\varepsilon}(y)= \begin{cases}I_{\varepsilon}^{\Omega}(y) & \text { if } y \in \mathcal{A}_{\varepsilon}^{\Omega}, \\ +\infty & \text { otherwise in } L^{p}\left(\Omega ; \mathbb{R}^{3}\right),\end{cases}
$$


$\Gamma$-converges in the strong topology of $L^{p}\left(\Omega ; \mathbb{R}^{3}\right)$ to the functional

$$
J(y)= \begin{cases}\int_{\omega} Q W_{0}\left(D_{\alpha} y\right) \mathrm{d} x_{\alpha}-\int_{\omega} \mathrm{m}_{3} f \cdot y \mathrm{~d} x_{\alpha} & \text { if } y \in W_{\Gamma}^{1, p}\left(\omega ; \mathbb{R}^{3}\right), \\ +\infty & \text { otherwise in } L^{p}\left(\Omega ; \mathbb{R}^{3}\right) .\end{cases}
$$

Here

$$
W_{\Gamma}^{1, p}\left(\omega ; \mathbb{R}^{3}\right):=\left\{y \in W^{1, p}\left(\omega ; \mathbb{R}^{3}\right): y(x)=\left(x_{1}, x_{2}, 0\right) \text { on } \partial \omega\right\},
$$

$\mathrm{m}_{3} f\left(x_{\alpha}\right)=\int_{-1 / 2}^{1 / 2} f\left(x_{\alpha}, x_{3}\right) \mathrm{d} x_{3}$ denotes the integral mean value of $f$ with respect to the third variable and $Q W_{0}$ denotes the quasiconvex envelope of the continuous function

$$
W_{0}(\bar{F}):=\min \left\{W(\bar{F} \mid z): z \in \mathbb{R}^{3}\right\}, \quad \bar{F} \in M^{3 \times 2},
$$

which satisfies the same growth conditions as $W$. A similar result in the case of strings was previously obtained by Acerbi, Buttazzo and Percivale [1].

With the motivation already explained in the introduction we extend the functionals $I_{\varepsilon}^{\Omega}$ to the larger space $L_{w}^{\infty}\left(\Omega ; \mathcal{M}\left(M^{3 \times 3}\right)\right)$ by setting

$$
I_{\varepsilon}^{\mathcal{M}}(\mu)= \begin{cases}I_{\varepsilon}^{\Omega}(y) & \text { if } \exists y \in \mathcal{A}_{\varepsilon}^{\Omega}: \mu=\delta_{D y(\cdot)} \\ +\infty & \text { otherwise in } L_{w}^{\infty}\left(\Omega ; \mathcal{M}\left(M^{3 \times 3}\right)\right) .\end{cases}
$$

Let us remark that this functional is well defined because if $y_{1}, y_{2} \in \mathcal{A}_{\varepsilon}^{\Omega}$ are such that $\delta_{D y_{1}(x)}=$ $\delta_{D y_{2}(x)}$ then, due to the boundary condition, $y_{1}=y_{2}$ almost everywhere.

\section{The limit problem}

Let us denote by $\bar{\pi}$ and $\pi^{3}$ the projections of $M^{3 \times 3}$ onto $M^{3 \times 2}$ and $\mathbb{R}^{3}$, respectively, that is,

$$
\begin{aligned}
\bar{\pi}: M^{3 \times 3} & \rightarrow M^{3 \times 2}, & \bar{\pi}(\bar{F} \mid z) & =\bar{F}, \\
\pi^{3}: M^{3 \times 3} & \rightarrow \mathbb{R}^{3}, & \pi^{3}(\bar{F} \mid z) & =z,
\end{aligned}
$$

and by $\bar{\pi}_{\#} \mu_{x}$ and $\pi_{\#}^{3} \mu_{x}$ the usual image measures (see Section 2).

We further denote by $\mathcal{Y}_{\Gamma}^{1, p}\left(\Omega ; \mathbb{R}^{3}\right)$ the set of $W^{1, p}\left(\Omega ; \mathbb{R}^{3}\right)$-gradient Young measures with an underlying deformation $y$ with $y(x)=\left(x_{1}, x_{2}, 0\right)$ on $\Gamma$, and by $\dot{\mathcal{Y}}_{\Gamma}^{1, p}\left(\Omega ; \mathbb{R}^{3}\right)$ the subset of $\mathcal{Y}_{\Gamma}^{1, p}\left(\Omega ; \mathbb{R}^{3}\right)$ of Young measures $\mu$ with $\pi_{\#}^{3} \mu=\delta_{0}$, that is,

$$
\dot{\mathcal{Y}}_{\Gamma}^{1, p}\left(\Omega ; \mathbb{R}^{3}\right):=\left\{\mu \in \mathcal{Y}_{\Gamma}^{1, p}\left(\Omega ; \mathbb{R}^{3}\right): \pi_{\#}^{3} \mu=\delta_{0}\right\} .
$$

The following lemma states that the sequence of functionals $I_{\varepsilon}^{\mathcal{M}}$ is equi-coercive in $\dot{\mathcal{Y}}_{\Gamma}^{1, p}\left(\Omega ; \mathbb{R}^{3}\right)$ with the weak* convergence of $L_{w}^{\infty}\left(\Omega ; \mathcal{M}\left(M^{3 \times 3}\right)\right)$.

LeMma 5.1 Let $\varepsilon_{n} \rightarrow 0$ and $\mu^{n} \in L_{w}^{\infty}\left(\Omega ; \mathcal{M}\left(M^{3 \times 3}\right)\right)$ be such that

$$
\sup _{n} I_{\varepsilon_{n}}^{\mathcal{M}}\left(\mu^{n}\right)<+\infty .
$$


Then there exists $\mu \in \dot{\mathcal{Y}}_{\Gamma}^{1, p}\left(\Omega ; \mathbb{R}^{3}\right)$ and a subsequence $\left(\mu^{n_{k}}\right)$ such that

$$
\mu^{n_{k}} \rightarrow \mu \quad \text { weak }^{*} \text { in } L_{w}^{\infty}\left(\Omega ; \mathcal{M}\left(M^{3 \times 3}\right)\right)
$$

Moreover, if $y^{n_{k}}$ and $y$ are the underlying deformations of $\mu^{n_{k}}$ and $\mu$ which satisfy the boundary conditions $y^{n_{k}}(x)=\left(x_{1}, x_{2}, \varepsilon_{n_{k}} x_{3}\right)$ and $y(x)=\left(x_{1}, x_{2}, 0\right)$, respectively, then

$$
y^{n_{k}} \rightarrow y \quad \text { weakly in } W^{1, p}\left(\Omega ; \mathbb{R}^{3}\right), \quad D_{3} y=0 .
$$

Proof. Since $\sup _{n} I_{\varepsilon_{n}}^{\mathcal{M}}\left(\mu^{n}\right)<+\infty$, we have $\mu_{x}^{n}=\delta_{D y^{n}(x)}, y^{n} \in W^{1, p}\left(\Omega ; \mathbb{R}^{3}\right)$ and $y^{n}(x)=$ $\left(x_{1}, x_{2}, \varepsilon_{n} x_{3}\right)$ on $\Gamma$. By the growth assumption on $W$ and the Poincaré inequality, for every $n$ large enough so that $\varepsilon_{n}<1$ we get

$$
\begin{aligned}
I_{\varepsilon_{n}}^{\mathcal{M}}\left(\mu^{n}\right) & =\int_{\Omega} W\left(D_{\alpha} y^{n} \mid \frac{D_{3} y^{n}}{\varepsilon_{n}}\right) \mathrm{d} x-\int_{\Omega} f \cdot y^{n} \mathrm{~d} x \\
& \geqslant c\left\|\left(D_{\alpha} y^{n} \mid \frac{D_{3} y^{n}}{\varepsilon_{n}}\right)\right\|_{p}^{p}-\|f\|_{q}\left\|y^{n}\right\|_{p}-c|\Omega| \\
& \geqslant c\left\|D y^{n}\right\|_{p}^{p}-\|f\|_{q}\left\|y^{n}\right\|_{p}-c|\Omega| \geqslant C_{1}\left\|y^{n}\right\|_{1, p}^{p}-C_{2},
\end{aligned}
$$

where $\|\cdot\|_{p}$ and $\|\cdot\|_{1, p}$ denote the usual $L^{p}$ and $W^{1, p}$ norms, respectively. Therefore

$$
\sup _{n}\left\|y^{n}\right\|_{1, p}<+\infty, \quad \sup _{n}\left\|\left(D_{\alpha} y^{n} \mid \frac{D_{3} y^{n}}{\varepsilon_{n}}\right)\right\|_{p}<+\infty,
$$

and thus, up to subsequences,

$$
y^{n} \rightarrow y \quad \text { weakly in } W^{1, p}\left(\Omega ; \mathbb{R}^{3}\right), \quad D_{3} y^{n} \rightarrow 0 \quad \text { strongly in } L^{p}\left(\Omega ; \mathbb{R}^{3}\right)
$$

and

$$
\mu^{n} \rightarrow \mu \quad \text { weak }^{*} \text { in } L_{w}^{\infty}\left(\Omega ; \mathcal{M}\left(M^{3 \times 3}\right)\right)
$$

It follows that $y=y\left(x_{1}, x_{2}\right)$ and $y(x)=\left(x_{1}, x_{2}, 0\right)$ on $\Gamma$ and $\pi_{\#}^{3} \mu=\delta_{0}$. Hence $\mu \in \dot{\mathcal{Y}}_{\Gamma}^{1, p}$.

If $\mu \in \dot{\mathcal{Y}}_{\Gamma}^{1, p}\left(\Omega ; \mathbb{R}^{3}\right)$ then it can be proved (see Lemma 5.3 below) that the center of mass of $\mu_{x}$ does not depend on the $x_{3}$ variable. With an example we are going to show that, in general, $\mu_{x}$ does depend on $x_{3}$. This is the main motivation which will lead us to study the $\Gamma$-convergence with respect to the weak* convergence of the averages with respect to the third variable.

LEMMA 5.2 If $\mu$ is a $W^{1, p}\left(\Omega ; \mathbb{R}^{3}\right)$-gradient Young measure generated by the sequence of gradients $D y^{\varepsilon}$, and $\nu$ and $\gamma$ are the Young measures generated by $D_{\alpha} y^{\varepsilon}$ and $D_{3} y^{\varepsilon}$, respectively, then

$$
\bar{\pi}_{\#} \mu=v, \quad \pi_{\#}^{3} \mu=\gamma .
$$

Proof. The proof is a direct consequence of the definitions and of the classical integration formula with respect to an image measure. Indeed, since $D_{\alpha} y^{\varepsilon}=\bar{\pi}\left(D y^{\varepsilon}\right)$, for every $\varphi \in C_{0}\left(M^{3 \times 2}\right)$ and 
$g \in L^{1}(\Omega)$ we have

$$
\begin{aligned}
\int_{\Omega}\left\langle\bar{\pi}_{\#} \mu_{x}, \varphi\right\rangle g(x) \mathrm{d} x & =\int_{\Omega} \int_{M^{3 \times 2}} \varphi(\bar{F}) \mathrm{d} \bar{\pi}_{\#} \mu_{x}(\bar{F}) g(x) \mathrm{d} x \\
& =\int_{\Omega} \int_{M^{3 \times 3}} \varphi(\bar{\pi}(F)) \mathrm{d} \mu_{x}(F) g(x) \mathrm{d} x \\
& =\lim _{\varepsilon \rightarrow 0} \int_{\Omega} \varphi\left(\bar{\pi}\left(D y^{\varepsilon}\right) g(x) \mathrm{d} x\right. \\
& =\lim _{\varepsilon \rightarrow 0} \int_{\Omega} \varphi\left(D_{\alpha} y^{\varepsilon}\right) g(x) \mathrm{d} x=\int_{\Omega}\left\langle v_{x}, \varphi\right\rangle g(x) \mathrm{d} x .
\end{aligned}
$$

The other relation can be proved similarly.

LEMMA 5.3 If $\mu \in L_{w}^{\infty}\left(\Omega ; \mathcal{M}\left(M^{3 \times 3}\right)\right)$, with $\pi_{\#}^{3} \mu=\delta_{0}$, is generated by the sequence of gradients $D y^{\varepsilon}$ with underlying deformation $y$, and $v$ is the Young measure generated by $D_{\alpha} y^{\varepsilon}$, then

$$
\mu=v \otimes \delta_{0} .
$$

Moreover, the function $y$ and the center of mass of the measures $\mu_{x}$ and $v_{x}$, for almost every $x \in \Omega$, do not depend on the variable $x_{3}$. In particular, with a small abuse of notation, we shall write

$$
\left\langle\mu_{x}, \mathrm{id}\right\rangle=D y\left(x_{\alpha}\right), \quad\left\langle v_{x}, \mathrm{id}\right\rangle=D_{\alpha} y\left(x_{\alpha}\right) .
$$

Proof. From $\pi_{\#}^{3} \mu=\delta_{0}$ and the previous lemma, we deduce that $D_{3} y^{\varepsilon}$ generates the Young measure $\delta_{0}$. The first part of the statement follows by applying Theorem 2.1 Moreover, we have

$$
D y(x)=\left\langle\mu_{x}, \mathrm{id}\right\rangle=\left\langle v_{x} \otimes \delta_{0}, \mathrm{id}\right\rangle=\int_{M^{3 \times 3}}(\bar{F} \mid z) \mathrm{d} v_{x} \otimes \delta_{0}(\bar{F} \mid z)=\left(\left\langle v_{x}, \mathrm{id}\right\rangle \mid 0\right)
$$

for almost every $x \in \Omega$. Thus $D_{3} y=0$, hence $y=y\left(x_{\alpha}\right)$ and $\left\langle v_{\left(x_{\alpha}, x_{3}\right)}, \mathrm{id}\right\rangle=D_{\alpha} y\left(x_{\alpha}\right)$.

EXAMPLE 5.4 We provide an example of a $\mu \in \dot{\mathcal{Y}}_{\Gamma}^{1, p}\left(\Omega ; \mathbb{R}^{3}\right)$ such that $\mu_{x}$ depends on $x_{3}$.

Let $\rho(s)$ be the 2-periodic function equaling $s$ on $[0,1]$ and $2-s$ on $[1,2]$. Let $z^{\varepsilon}(x):=$ $\left(\varepsilon x_{3} \rho\left(x_{1} / \varepsilon\right), 0,0\right)$. Then $D_{3} z^{\varepsilon} \rightarrow 0$ in $L^{\infty}\left(\Omega ; \mathbb{R}^{3}\right)$. Moreover, since

$$
D_{1} z_{1}^{\varepsilon}(x)=x_{3} \rho^{\prime}\left(\frac{x_{1}}{\varepsilon}\right) \text { generates the Young measure } \frac{1}{2} \delta_{-x_{3}}+\frac{1}{2} \delta_{x_{3}},
$$

it follows that

$$
D_{\alpha} z^{\varepsilon} \text { generates the Young measure } \frac{1}{2} \delta_{-x_{3} \bar{E}^{11}}+\frac{1}{2} \delta_{x_{3} \bar{E}^{11}},
$$

where $\bar{E}^{11}$ is the matrix in $M^{3 \times 2}$ with 1 in position 11 and all other elements zero. It is now easy to check that the functions $y^{\varepsilon}(x):=\left(x_{1}, x_{2}, \varepsilon x_{3}\right)+z^{\varepsilon}(x) \min \{1$ dist $(x, \partial \Omega) / \varepsilon\}$ are in $\mathcal{A}_{\varepsilon}^{\Omega}$ and they generate a $\mu \in \dot{\mathcal{Y}}_{\Gamma}^{1, p}$ which depends also on $x_{3}$.

The example above motivates the following definition. 
Definition 5.5 Let $X$ be either $M^{3 \times 2}$ or $M^{3 \times 3}$. If $v \in L_{w}^{\infty}(\Omega ; \mathcal{M}(X))$ we define

$$
A v^{3} v: \omega \rightarrow \mathcal{M}(X),
$$

the average of $v$ with respect to the variable $x_{3}$, by

$$
\left\langle\mathrm{Av}_{x_{\alpha}}^{3} \nu, \varphi\right\rangle:=\left\langle\operatorname{Av}^{3} v\left(x_{\alpha}\right), \varphi\right\rangle:=\int_{-1 / 2}^{1 / 2}\left\langle v_{\left(x_{\alpha}, x_{3}\right)}, \varphi\right\rangle \mathrm{d} x_{3}
$$

for every $\varphi \in C_{0}(X)$.

By Fubini's theorem the map $x_{\alpha} \mapsto\left\langle\mathrm{Av}_{x_{\alpha}}^{3} \nu, \varphi\right\rangle$ is measurable, and since it is also essentially bounded, we can think of $\mathrm{Av}^{3}$ as a mapping

$$
\operatorname{Av}^{3}: L_{w}^{\infty}(\Omega ; \mathcal{M}(X)) \rightarrow L_{w}^{\infty}(\omega ; \mathcal{M}(X))
$$

It is easy to check that this map is continuous, that is,

$$
\begin{array}{rc}
\mu^{\varepsilon} \rightarrow \mu & \text { weak }^{*} \text { in } L_{w}^{\infty}(\Omega ; \mathcal{M}(X)) \\
& \Downarrow \\
\mathrm{Av}^{3} \mu^{\varepsilon} \rightarrow \mathrm{Av}^{3} \mu & \text { weak }^{*} \text { in } L_{w}^{\infty}(\omega ; \mathcal{M}(X)),
\end{array}
$$

and that $\mathrm{Av}^{3}$ maps Young measures to Young measures.

The $\Gamma$-convergence result will be made more expressive by using the average-projection mapping

defined by

$$
q: L_{w}^{\infty}\left(\Omega ; \mathcal{M}\left(M^{3 \times 3}\right)\right) \rightarrow L_{w}^{\infty}\left(\omega ; \mathcal{M}\left(M^{3 \times 2}\right)\right),
$$

$$
q=\bar{\pi}_{\#} \circ \mathrm{Av}^{3}=\mathrm{Av}^{3} \circ \bar{\pi}_{\#},
$$

where the commutativity of composition comes directly from the definitions. By the continuity of the average and the projection mapping, even $q$ is continuous.

We are now in a position to state the main results of this section, whose proofs are postponed to Section 8

We denote by $\mathcal{Y}_{\Gamma}^{1, p}\left(\omega ; \mathbb{R}^{3}\right)$ the subset of $W^{1, p}\left(\omega ; \mathbb{R}^{3}\right)$-gradient Young measures with an underlying deformation $y$ with $y(x)=\left(x_{1}, x_{2}, 0\right)$ on $\partial \omega$.

The following theorems hold under the continuity and growth assumptions made on $W$ at the beginning of Section 4

THEOREM 5.6 The family of energy functionals $I_{s}^{\mathcal{M}}$, already defined in (4.4), is $\left(q, w^{*} L_{w}^{\infty}\left(\omega ; \mathcal{M}\left(M^{3 \times 2}\right)\right)\right)$-equi-coercive (see Definition 3.3 .

THEOREM 5.7 Let $I_{\varepsilon}^{\mathcal{M}}$ be the family of functionals defined in (4.4). Then

$$
\Gamma\left(q, w^{*} L_{w}^{\infty}\left(\omega ; \mathcal{M}\left(M^{3 \times 2}\right)\right)\right) \lim _{\varepsilon \rightarrow 0} I_{\varepsilon}^{\mathcal{M}}(v)=I(v)
$$

with

$$
I(v)= \begin{cases}\int_{\omega}\left\langle v_{x_{\alpha}}, W_{0}\right\rangle \mathrm{d} x_{\alpha}-\int_{\omega} \mathrm{m}_{3} f \cdot y \mathrm{~d} x_{\alpha} & \text { if } v \in \mathcal{Y}_{\Gamma}^{1, p}\left(\omega ; \mathbb{R}^{3}\right) \\ +\infty & \text { otherwise in } L_{w}^{\infty}\left(\omega ; \mathcal{M}\left(M^{3 \times 2}\right)\right)\end{cases}
$$


where $W_{0}$ is the function defined in (4.3), and $y \in W^{1, p}\left(\omega ; \mathbb{R}^{3}\right)$ is the underlying deformation of $v$ with boundary condition $y=\left(x_{1}, x_{2}, 0\right)$ on $\partial \omega$.

By the properties of $\Gamma$-limits summarized in Proposition 3.4 we can state the following theorem which makes precise the variational character of the limit energy $I(v)$.

THEOREM 5.8 Let $I$ be the functional defined in Theorem 5.7 We have:

(i) $I$ is weak* lower semicontinuous and weak* coercive; hence it admits a minimum on the space $\mathcal{Y}_{\Gamma}^{1, p}\left(\omega ; \mathbb{R}^{3}\right) ;$

(ii) if $y^{\varepsilon} \in W^{1, p}\left(\Omega ; \mathbb{R}^{3}\right)$ satisfies the boundary condition $y^{\varepsilon}(x)=\left(x_{1}, x_{2}, \varepsilon x_{3}\right)$ on $\Gamma$ and $\liminf _{\varepsilon \rightarrow 0} I_{\varepsilon}^{\Omega}\left(y^{\varepsilon}\right)=\liminf _{\varepsilon \rightarrow 0} \inf I_{\varepsilon}^{\Omega}$ (e.g. if $y^{\varepsilon}$ minimizes $\left.I_{\varepsilon}^{\Omega}\right)$ then

(a) if $\varepsilon_{n} \rightarrow 0$ and if $q\left(\delta_{D y^{\varepsilon_{n}}}\right) \rightarrow v$ weak* in $L_{w}^{\infty}\left(\omega ; \mathcal{M}\left(M^{3 \times 2}\right)\right)$ then $v$ is a minimizer of $I$ on $\mathcal{Y}_{\Gamma}^{1, p}\left(\omega ; \mathbb{R}^{3}\right)$ and $\lim _{n \rightarrow \infty} I_{\varepsilon_{n}}^{\Omega}\left(y^{\varepsilon_{n}}\right)=I(v)$;

(b) there is a sequence $\varepsilon_{n} \rightarrow 0$ and a minimizer $v$ of $I$ on $\mathcal{Y}_{\Gamma}^{1, p}\left(\omega ; \mathbb{R}^{3}\right)$ such that $q\left(\delta_{D y_{n}^{\varepsilon}}\right) \rightarrow v$ weak* in $L_{w}^{\infty}\left(\omega ; \mathcal{M}\left(M^{3 \times 2}\right)\right)$.

\section{Relationship with Le Dret and Raoult theory}

Let $J$ be the $\Gamma$-limit functional of Le Dret and Raoult introduced in 4.2 .

THEOREM 6.1 Let $y \in L^{p}\left(\Omega ; \mathbb{R}^{3}\right)$. Then

$$
J(y)=\inf I(v),
$$

where the infimum is taken over all $v \in L_{w}^{\infty}\left(\omega ; \mathcal{M}\left(M^{3 \times 2}\right)\right)$ which satisfy $\langle v$, id $\rangle=D_{\alpha} y$ and $y(x)=\left(x_{1}, x_{2}, 0\right)$ on $\partial \omega$, with the usual convention $\inf \emptyset=+\infty$. Moreover the infimum is attained.

Proof. Let $y \in L^{p}\left(\Omega ; \mathbb{R}^{3}\right)$. Let us first of all observe that the infimum is attained as a consequence of item (i) of Theorem 5.8 and the continuity of the center of mass with respect to the weak* convergence in $L_{w}^{\infty}\left(\omega ; \mathcal{M}\left(M^{3 \times 2}\right)\right)$.

For convenience we denote the right hand side of 6.1 by $\widetilde{I}(y)$.

Let us start by proving the inequality $J(y) \leqslant \widetilde{I}(y)$. To this end we can assume that $\widetilde{I}(y)$ is finite, that is, there exists $v \in L_{w}^{\infty}\left(\omega ; \mathcal{M}\left(M^{3 \times 2}\right)\right)$ such that $I(v)=\widetilde{I}(y)$ and $y$ satisfies the boundary value problem $D_{\alpha} y=\langle\nu$, id $\rangle, y(x)=\left(x_{1}, x_{2}, 0\right)$ on $\Gamma$. Hence $y=y\left(x_{\alpha}\right)$. Then, since $I(v)<+\infty$, we have $v \in \mathcal{Y}_{\Gamma}^{1, p}\left(\omega ; \mathbb{R}^{3}\right)$ and therefore $y \in W_{\Gamma}^{1, p}\left(\omega ; \mathbb{R}^{3}\right)$. In this case

$$
J(y)=\int_{\omega} Q W_{0}\left(D_{\alpha} y\right) \mathrm{d} x_{\alpha}-\int_{\omega} \mathrm{m}_{3} f \cdot y \mathrm{~d} x_{\alpha},
$$

and, from (iii) of Theorem 2.5, we have

$$
Q W_{0}\left(D_{\alpha} y\right) \leqslant\left\langle\nu, W_{0}\right\rangle,
$$

which implies that $J(y) \leqslant I(v)=\widetilde{I}(y)$.

In order to prove the converse inequality, it is convenient to use the fact that the functionals involved are $\Gamma$-limits. As

$$
J(y)=\Gamma^{-}\left(L^{p}\left(\Omega ; \mathbb{R}^{3}\right)\right) \lim _{\varepsilon \rightarrow 0} J_{\varepsilon}(y),
$$


there exist sequences $\varepsilon_{n} \rightarrow 0$ and $y^{n} \rightarrow y$ weakly in $W^{1, p}\left(\Omega ; \mathbb{R}^{3}\right)$ such that

$$
\lim _{n \rightarrow \infty} J_{\varepsilon_{n}}\left(y^{n}\right)=J(y) .
$$

Since the only interesting case is $J(y)<+\infty$, by coercivity and possibly passing to subsequences, we can assume that

- $\left(D y^{n}\right)$ generates a $W^{1, p}\left(\Omega ; \mathbb{R}^{3}\right)$-Young measure $\mu$ with underlying deformation $y$,

- $\left(D_{\alpha} y^{n}\right)$ generates a Young measure $v_{\Omega}$,

- $D_{3} y^{n} \rightarrow D_{3} y=0$ strongly in $L^{p}$, hence $D_{3} y_{n}$ generates the measure $\delta_{0}$.

By Lemma 5.2 it follows that $\bar{\pi}_{\#} \mu=v_{\Omega}$ and $\pi_{\#}^{3} \mu=\delta_{0}$, so $\mu \in \dot{\mathcal{Y}}_{\Gamma}^{1, p}\left(\Omega ; \mathbb{R}^{3}\right)$ by definition. An application of Lemma 5.3 gives $\mu=v_{\Omega} \otimes \delta_{0}$ and $\left\langle v_{\Omega}\right.$, id $\rangle=D_{\alpha} y$. Let $\mu^{n}=\delta_{D y^{n}}$ and $v:=$ $q(\mu)=\operatorname{Av}^{3}\left(v_{\Omega}\right)$. Then $v \in \mathcal{Y}_{\Gamma}^{1, p}\left(\omega ; \mathbb{R}^{3}\right)$ and $\langle v$, id $\rangle=D_{\alpha} y$. From the continuity of $q$,

$$
q\left(\mu^{n}\right) \rightarrow v \quad \text { weak }{ }^{*} \text { in } L_{w}^{\infty}\left(\omega ; \mathcal{M}\left(M^{3 \times 2}\right)\right)
$$

and by the $\Gamma$-convergence theorem 5.7 and the fact that $J_{\varepsilon_{n}}\left(y_{n}\right)=I_{\varepsilon_{n}}^{\mathcal{M}}\left(\mu^{n}\right)$, we obtain

$$
J(y)=\lim _{n \rightarrow \infty} J_{\varepsilon_{n}}\left(y^{n}\right)=\liminf _{n \rightarrow \infty} I_{\varepsilon_{n}}^{\mathcal{M}}\left(\mu^{n}\right) \geqslant I(v) \geqslant \widetilde{I}(y),
$$

which concludes the proof.

The relationship between the minimizers of the two functionals $I$ and $J$ is made explicit in the following theorem.

THEOREM 6.2 The following properties hold for the functionals $I$ and $J$.

(i) $\min \left\{J(y): y \in W_{\Gamma}^{1, p}\left(\omega ; \mathbb{R}^{3}\right)\right\}=\min \left\{I(\nu): v \in \mathcal{Y}_{\Gamma}^{1, p}\left(\omega ; \mathbb{R}^{3}\right)\right\}$.

(ii) Let $v$ be a minimizer for $I$. If $y \in W_{\Gamma}^{1, p}\left(\omega ; \mathbb{R}^{3}\right)$ satisfies $D_{\alpha} y\left(x_{\alpha}\right)=\left\langle v_{x_{\alpha}}\right.$, id $\rangle$ for almost every $x_{\alpha} \in \omega$, then $y$ is a minimizer for $J$ and $Q W_{0}\left(D_{\alpha} y\left(x_{\alpha}\right)\right)=\left\langle v_{x_{\alpha}}, W_{0}\right\rangle$ for almost every $x_{\alpha} \in \omega$.

(iii) Let $y$ be a minimizer for $J$. If $v \in \mathcal{Y}_{\Gamma}^{1, p}\left(\omega ; \mathbb{R}^{3}\right)$ satisfies $D_{\alpha} y\left(x_{\alpha}\right)=\left\langle v_{x_{\alpha}}\right.$,id $\rangle$ and $Q W_{0}\left(D_{\alpha} y\left(x_{\alpha}\right)\right)=\left\langle v_{x_{\alpha}}, W_{0}\right\rangle$ for almost every $x_{\alpha} \in \omega$, then $v$ is a minimizer for $I$.

Proof. This follows by noticing that $J$ and $I$ are the relaxed functionals of the extension by $+\infty$ of

$$
E(y)=\int_{\omega} W_{0}\left(D_{\alpha} y\right) \mathrm{d} x_{\alpha}-\int_{\omega} \mathrm{m}_{3} f \cdot y \mathrm{~d} x_{\alpha}
$$

with respect to the strong topology of $L^{p}\left(\omega ; \mathbb{R}^{3}\right)$ and the weak topology of the space $L_{w}^{\infty}\left(\omega ; \mathcal{M}\left(M^{3 \times 2}\right)\right)$, respectively, and applying Theorem 4.4 and Corollary 4.6 of Pedregal [27].

\section{The energy density of the thin film}

The aim of this section is to propose and motivate an energy density for the thin film. Indeed we shall deduce that $W_{0}$ and

$$
E(y)=\int_{\omega} W_{0}\left(D_{\alpha} y\right) \mathrm{d} x_{\alpha}-\int_{\omega} \mathrm{m}_{3} f \cdot y \mathrm{~d} x_{\alpha}
$$

are the energy density and the total energy of the thin film, respectively. 
We need to work with a general linear boundary condition $y(x)=B x$ on $\Gamma_{\varepsilon}$, where $B$ is a linear map from $\mathbb{R}^{3}$ into itself. The right Sobolev space to treat this case is

$$
W_{\Gamma_{B}}^{1, p}\left(\omega ; \mathbb{R}^{3}\right):=\left\{y \in W^{1, p}\left(\omega ; \mathbb{R}^{3}\right): y\left(x_{\alpha}\right)=\bar{B} x_{\alpha} \text { on } \partial \omega\right\}
$$

where $\bar{B} \in M^{3 \times 2}$ denotes the first two columns of the matrix representing $B$. It is trivial to check that in this setting the variational limit is the functional $I$ defined in 5.1$)$ with $\mathcal{Y}_{\Gamma}^{1, p}\left(\omega ; \mathbb{R}^{3}\right)$ replaced, with the obvious meaning of the notation, by $\mathcal{Y}_{\Gamma_{B}}^{1, p}\left(\omega ; \mathbb{R}^{3}\right)$, and the corresponding limit functional is denoted by $I_{B}$. Similarly, also the case studied by Le Dret and Raoult can be put into this framework and the limit functional is defined on $W_{\Gamma_{B}}^{1, p}\left(\omega ; \mathbb{R}^{3}\right)$ and denoted by $J_{B}$.

For every continuous function $Z: M^{3 \times 2} \rightarrow \mathbb{R}$ satisfying the growth condition of order $p$, $c\left(|\bar{F}|^{p}-1\right) \leqslant Z(\bar{F}) \leqslant C\left(1+|\bar{F}|^{p}\right)$ for $0<c<C$, we introduce the energy

$$
E_{Z}(y)=\int_{\omega} Z\left(D_{\alpha} y\right) \mathrm{d} x_{\alpha}-\int_{\omega} \mathrm{m}_{3} f \cdot y \mathrm{~d} x_{\alpha}
$$

and denote by $Q\left[E_{Z}, \Gamma_{B}\right]$ the relaxed functional, that is, the lower semicontinuous envelope of $E_{Z}$ on the space $W_{\Gamma_{B}}^{1, p}\left(\omega ; \mathbb{R}^{3}\right)$ with respect to the weak topology. By $Y\left[E_{Z}, \Gamma_{B}\right]$ we mean the relaxation of

$$
E_{Z}^{\infty}(v)= \begin{cases}E_{Z}(y) & \text { if } v=\delta_{D_{\alpha} y}, y \in W_{\Gamma_{B}}^{1, p}\left(\omega ; \mathbb{R}^{3}\right), \\ +\infty & \text { otherwise in } L_{w}^{\infty}\left(\omega ; \mathcal{M}\left(M^{3 \times 2}\right)\right),\end{cases}
$$

with respect to the weak* topology of $L_{w}^{\infty}\left(\omega ; \mathcal{M}\left(M^{3 \times 2}\right)\right)$.

As briefly noticed in the last proof, the limit functional $J_{B}$, obtained by Le Dret and Raoult, and the functional $I_{B}$, obtained by us, can be written (see Dacorogna [16] and, for instance, Murat and Tartar [25]) as

$$
J_{B}(y)=Q\left[E_{W_{0}}, \Gamma_{B}\right](y), \quad I_{B}(v)=Y\left[E_{W_{0}}, \Gamma_{B}\right](v) .
$$

THEOREM 7.1 The function $W_{0}$ is the unique continuous integrand satisfying a growth condition of order $p$ such that $I_{B}=Y\left[E_{W_{0}}, \Gamma_{B}\right]$ for every linear boundary condition, that is, for every linear $\operatorname{map} B$.

Proof. Let $W_{1}$ be one of these functions such that $I_{B}=Y\left[E_{W_{1}}, \Gamma_{B}\right]$ for every linear $B$. Then

$$
\int_{\omega}\left\langle v_{x_{\alpha}}, W_{0}-W_{1}\right\rangle \mathrm{d} x_{\alpha}=0 \quad \text { for every } v \in \mathcal{Y}_{\Gamma_{B}}^{1, p}\left(\omega ; \mathbb{R}^{3}\right)
$$

and for every $B$. It follows that $W_{0}=W_{1}$ by simply taking the admissible measure $v=\delta_{\bar{B}}$, where $\bar{B} \in M^{3 \times 2}$ are the first two columns of the matrix representing $B$.

Hence, the variational problem $I$ determines a unique energy density $W_{0}$. This selection is not provided by the relation $J_{B}=Q\left[W_{0}, \Gamma_{B}\right]$; indeed, in general, there exist an infinite number of functions $W_{1}$ such that $J_{B}=Q\left[W_{0}, \Gamma_{B}\right]=Q\left[W_{1}, \Gamma_{B}\right]$ for every $B$.

A further reason to take $W_{0}$ as the energy density of the thin film follows from the relation between $E=E_{W_{0}}$ and $I$, which by the well known properties of relaxed functionals can be expressed in terms of correspondence between minimizing sequences of $E$ and minimizers of $I$. In particular, minimizing sequences of $E$ generate Young measures which are minimizers of $I$, and vice versa.

In Le Dret and Raoult [22] it is shown that if the energy $W$ satisfies the principle of material frame indifference, then also $W_{0}$ is frame indifferent, and moreover the symmetry relations of $W_{0}$ are derived from those of $W$. 


\section{Proofs of the main results of Section 5}

Before proving the main theorems, we state some technical results.

LEMmA 8.1 Let $v \otimes \delta_{0}$ be a $W^{1, p}\left(\Omega ; \mathbb{R}^{3}\right)$-gradient Young measure. Then $A v^{3} v$ is a $W^{1, p}\left(\omega ; \mathbb{R}^{3}\right)$ gradient Young measure, and $\left\langle\mathrm{Av}_{x_{\alpha}}^{3} \nu, \mathrm{id}\right\rangle=\left\langle v_{\left(x_{\alpha}, x_{3}\right)}\right.$, id $\rangle$ for almost every $x \in \Omega$. Moreover, for every $W^{1, p}\left(\omega ; \mathbb{R}^{3}\right)$-gradient Young measure $\bar{\mu}$, there exists a $W^{1, p}\left(\Omega ; \mathbb{R}^{3}\right)$-gradient Young measure $\mu \otimes \delta_{0}$ such that $\bar{\mu}=\mathrm{Av}^{3} \mu$.

Proof. To show that $A v^{3} v$ is a $W^{1, p}\left(\omega ; \mathbb{R}^{3}\right)$-gradient Young measure we may use the characterization given by Kinderlehrer and Pedregal (see Theorem 2.5). Thus it suffices to show that

(i) $\int_{\omega} \int_{M^{3 \times 2}}|\bar{F}|^{p} \mathrm{dAv}_{x_{\alpha}}^{3} v(\bar{F}) \mathrm{d} x_{\alpha}<+\infty$;

(ii) $\left\langle\mathrm{Av}_{x_{\alpha}}^{3} \nu\right.$, id $\rangle=D_{\alpha} y\left(x_{\alpha}\right)$ and $y \in W^{1, p}\left(\omega ; \mathbb{R}^{3}\right)$;

(iii) $\left\langle\mathrm{Av}_{x_{\alpha}}^{3} \nu, f\right\rangle \geqslant f\left(\left\langle\mathrm{Av}_{x_{\alpha}}^{3} \nu, \mathrm{id}\right\rangle\right)$ for almost every $x_{\alpha}$ and all quasiconvex functions $f$ with $|f|(\bar{F}) \leqslant C\left(|\bar{F}|^{p}+1\right)$.

Property (i) holds since

$$
\begin{aligned}
\int_{\omega} \int_{M^{3 \times 2}}|\bar{F}|^{p} \mathrm{dAv}_{x_{\alpha}}^{3} \nu(\bar{F}) \mathrm{d} x_{\alpha} & =\int_{\omega} \int_{-1 / 2}^{1 / 2} \int_{M^{3 \times 2}}|\bar{F}|^{p} \mathrm{~d} v_{\left(x_{\alpha}, x_{3}\right)}(\bar{F}) \mathrm{d} x_{3} d x_{\alpha} \\
& =\int_{\Omega} \int_{M^{3 \times 2}}|(\bar{F} \mid z)|^{p} \mathrm{~d} v_{x} \otimes \delta_{0}(\bar{F} \mid z) \mathrm{d} x<+\infty .
\end{aligned}
$$

Before proving property (ii) let us note that if $y \in W^{1, p}\left(\Omega ; \mathbb{R}^{3}\right)$ is an underlying deformation for $v \otimes \delta_{0}$, then (see Lemma 5.3 $y=y\left(x_{\alpha}\right)$, and $\left\langle v_{\left(x_{\alpha}, x_{3}\right)}, \mathrm{id}\right\rangle=D_{\alpha} y\left(x_{\alpha}\right)$. It follows that

$$
\left\langle\operatorname{Av}_{x_{\alpha}}^{3} \nu, \mathrm{id}\right\rangle=\int_{-1 / 2}^{1 / 2}\left\langle v_{\left(x_{\alpha}, x_{3}\right)}, \mathrm{id}\right\rangle \mathrm{d} x_{3}=D_{\alpha} y\left(x_{\alpha}\right)
$$

hence property (ii) holds and $\left\langle\mathrm{Av}_{x_{\alpha}}^{3} \nu\right.$, id $\rangle=\left\langle v_{\left(x_{\alpha}, x_{3}\right)}\right.$, id $\rangle$. Let $f: M^{3 \times 2} \rightarrow \mathbb{R}$ be a quasiconvex function with $|f|(\bar{F}) \leqslant C\left(|\bar{F}|^{p}+1\right)$. That is, for every open and bounded set $U \subset \mathbb{R}^{2}$ with $|\partial U|=0$ one has

$$
\int_{U} f\left(\bar{F}+D_{\alpha} \varphi\right) \mathrm{d} x_{\alpha} \geqslant f(\bar{F})|U|
$$

for all $\varphi \in C_{0}^{1}\left(U ; \mathbb{R}^{3}\right)$ and all $\bar{F} \in M^{3 \times 2}$. Let $f^{\sharp}: M^{3 \times 3} \rightarrow \mathbb{R}$ be defined by $f^{\sharp}(\bar{F} \mid z):=f(\bar{F})$. Then for every $F=(\bar{F} \mid z) \in M^{3 \times 3}$ and $\psi \in C_{0}^{1}\left(U \times(-1 / 2,1 / 2) ; \mathbb{R}^{3}\right)$ we have

$$
\begin{aligned}
\int_{U \times(-1 / 2,1 / 2)} f^{\sharp}(F+D \psi) \mathrm{d} x & =\int_{-1 / 2}^{1 / 2} \int_{U} f\left(\bar{F}+D_{\alpha} \psi\left(x_{\alpha}, x_{3}\right)\right) \mathrm{d} x_{\alpha} d x_{3} \\
& \geqslant \int_{-1 / 2}^{1 / 2} f(\bar{F})|U| \mathrm{d} x_{3}=f^{\sharp}(\bar{F} \mid z)|U|=f^{\sharp}(F)|U|,
\end{aligned}
$$

where we have used the fact that $\psi\left(\cdot, x_{3}\right) \in C_{0}^{1}\left(U ; \mathbb{R}^{3}\right)$ for almost every $x_{3}$. Thus also $f^{\sharp}$ is quasi- 
convex. Hence, we have

$$
\begin{aligned}
\left\langle A v_{x_{\alpha}}^{3} \nu, f\right\rangle & =\int_{-1 / 2}^{1 / 2} \int_{M^{3 \times 2}} f(\bar{F}) \mathrm{d} v_{\left(x_{\alpha}, x_{3}\right)}(\bar{F}) \mathrm{d} x_{3}=\int_{-1 / 2}^{1 / 2} \int_{M^{3 \times 3}} f^{\sharp}(\bar{F} \mid z) \mathrm{d} v_{\left(x_{\alpha}, x_{3}\right)} \otimes \delta_{0}(\bar{F} \mid z) \mathrm{d} x_{3} \\
& =\int_{-1 / 2}^{1 / 2}\left\langle v_{\left(x_{\alpha}, x_{3}\right)} \otimes \delta_{0}, f^{\sharp}\right\rangle \mathrm{d} x_{3} \geqslant \int_{-1 / 2}^{1 / 2} f^{\sharp}\left(\left\langle v_{\left(x_{\alpha}, x_{3}\right)} \otimes \delta_{0}, \mathrm{id}\right\rangle\right) \mathrm{d} x_{3} \\
& =\int_{-1 / 2}^{1 / 2} f^{\sharp}\left(D_{\alpha} y\left(x_{\alpha}\right) \mid 0\right) \mathrm{d} x_{3}=f\left(D_{\alpha} y\left(x_{\alpha}\right)\right)=f\left(\left\langle A v_{x_{\alpha}}^{3} \nu, \mathrm{id}\right\rangle\right),
\end{aligned}
$$

which proves property (iii). In the last equality we have used 8.1 . We now prove the second part of the theorem. Let $\bar{\mu}$ be a $W^{1, p}\left(\omega ; \mathbb{R}^{3}\right)$-gradient Young measure. Then, obviously, $\bar{\mu} \otimes \delta_{0}$ is a $W^{1, p}\left(\Omega ; \mathbb{R}^{3}\right)$-gradient Young measure and $\mathrm{Av}^{3} \bar{\mu}=\bar{\mu}$.

The next lemma relates the spaces $\mathcal{Y}_{\Gamma}^{1, p}\left(\Omega ; \mathbb{R}^{3}\right)$ and $\mathcal{Y}_{\Gamma}^{1, p}\left(\omega ; \mathbb{R}^{3}\right)$.

LEMMA 8.2 We have

$$
\operatorname{Av}^{3} \dot{\mathcal{Y}}_{\Gamma}^{1, p}\left(\Omega ; \mathbb{R}^{3}\right)=\mathcal{Y}_{\Gamma}^{1, p}\left(\omega ; \mathbb{R}^{3}\right) \otimes \delta_{0}
$$

Proof. Let $\mu \in \dot{\mathcal{Y}}_{\Gamma}^{1, p}\left(\Omega ; \mathbb{R}^{3}\right)$. Then, by Lemma 5.3. $\mu=v \otimes \delta_{0}$, and for $\varphi \in C_{0}\left(M^{3 \times 3}\right)$ we have

$$
\begin{aligned}
\left\langle\operatorname{Av}_{x_{\alpha}}^{3} \mu, \varphi\right\rangle & =\int_{-1 / 2}^{1 / 2} \int_{M^{3 \times 3}} \varphi(\bar{F} \mid z) \mathrm{d} v_{\left(x_{\alpha}, x_{3}\right)} \otimes \delta_{0}(\bar{F} \mid z) \mathrm{d} x_{3}=\int_{-1 / 2}^{1 / 2} \int_{M^{3 \times 2}} \varphi(\bar{F} \mid 0) \mathrm{d} v_{\left(x_{\alpha}, x_{3}\right)}(\bar{F}) \mathrm{d} x_{3} \\
& =\int_{M^{3 \times 2}} \varphi(\bar{F} \mid 0) \mathrm{dAv}_{x_{\alpha}}^{3} v(\bar{F})=\int_{M^{3 \times 3}} \varphi(\bar{F} \mid z) \mathrm{dAv}_{x_{\alpha}}^{3} \nu \otimes \delta_{0}(\bar{F} \mid z)=\left\langle\left(\operatorname{Av}_{x_{\alpha}}^{3} \nu\right) \otimes \delta_{0}, \varphi\right\rangle
\end{aligned}
$$

Thus

$$
A v^{3}\left(v \otimes \delta_{0}\right)=\left(A v^{3} v\right) \otimes \delta_{0}
$$

and the assertion follows by an easy application of Lemma 8.1 .

REMARK 8.3 A useful consequence of Lemma 8.2 is

$$
q\left(\dot{\mathcal{Y}}_{\Gamma}^{1, p}\left(\Omega ; \mathbb{R}^{3}\right)\right)=\mathcal{Y}_{\Gamma}^{1, p}\left(\omega ; \mathbb{R}^{3}\right) .
$$

Proof of Theorem 5.6. Since $L_{w}^{\infty}\left(\omega ; \mathcal{M}\left(M^{3 \times 2}\right)\right)$ is the dual of the space $L^{1}\left(\omega ; C_{0}\left(M^{3 \times 2}\right)\right)$ which is Banach and separable, the proof is a direct consequence of the following proposition.

Proposition 8.4 If $\varepsilon_{n} \rightarrow 0$ and the sequence $\mu^{n} \in L_{w}^{\infty}\left(\Omega ; \mathcal{M}\left(M^{3 \times 3}\right)\right)$ satisfy

$$
\sup _{n} I_{\varepsilon_{n}}^{\mathcal{M}}\left(\mu^{n}\right)<+\infty
$$

then there exist $n_{k} \rightarrow \infty$ and a Young measure $v \in L_{w}^{\infty}\left(\omega ; \mathcal{M}\left(M^{3 \times 2}\right)\right)$ such that $q\left(\mu^{n_{k}}\right) \rightarrow v$ weak ${ }^{*}$ in $L_{w}^{\infty}\left(\omega ; \mathcal{M}\left(M^{3 \times 2}\right)\right)$. Moreover $v \in \mathcal{Y}_{\Gamma}^{1, p}\left(\omega ; \mathbb{R}^{3}\right)$.

Proof. By Lemma 5.1 there exists $\mu \in \dot{\mathcal{Y}}_{\Gamma}^{1, p}\left(\Omega ; \mathbb{R}^{3}\right)$ and a subsequence $\mu^{n_{k}}$ such that

$$
\mu^{n_{k}} \rightarrow \mu \quad \text { weak }^{*} \text { in } L_{w}^{\infty}\left(\Omega ; \mathcal{M}\left(M^{3 \times 3}\right)\right)
$$


Let $v=q(\mu)$. By continuity of $q$ we conclude that $q\left(\mu^{n_{k}}\right) \rightarrow v$ weak ${ }^{*}$ in $L_{w}^{\infty}\left(\omega ; \mathcal{M}\left(M^{3 \times 2}\right)\right)$. Moreover, by Remark $8.3, v \in \mathcal{Y}_{\Gamma}^{1, p}\left(\omega ; \mathbb{R}^{3}\right)$.

Proof of Theorem 5.7. Let $\varepsilon_{n}$ be an infinitesimal sequence of positive real numbers. Since the sequence of functionals $I_{\varepsilon_{n}}^{\mathcal{M}}$ is $\left(q, w^{*} L_{w}^{\infty}\left(\omega ; \mathcal{M}\left(M^{3 \times 2}\right)\right)\right)$-equi-coercive, Proposition 3.5 applies. We start by finding a recovering sequence for a suitable subsequence $I_{\varepsilon_{n_{j}}}^{\mathcal{M}}$; that is, for $v \in \mathcal{Y}_{\Gamma}^{1, p}\left(\omega ; \mathbb{R}^{3}\right)$ we find a sequence $y^{j} \in W^{1, p}\left(\Omega ; \mathbb{R}^{3}\right)$ with $y^{j}(x)=\left(x_{1}, x_{2}, \varepsilon_{n_{j}} x_{3}\right)$ on $\Gamma$ such that the projections on the first two columns of the averages with respect to $x_{3}$ of the associated Young measures $\mu^{j}=\delta_{D y^{j}}$ converge to $\nu$, weak ${ }^{*}$ in $L_{w}^{\infty}\left(\omega ; \mathcal{M}\left(M^{3 \times 2}\right)\right)$, and

$$
\limsup _{j \rightarrow \infty} I_{\varepsilon_{n_{j}}}^{\mathcal{M}}\left(\mu^{j}\right) \leqslant I(v) .
$$

Let $y \in W^{1, p}\left(\omega ; \mathbb{R}^{3}\right)$ be the underlying deformation of $v$ with $y=\left(x_{1}, x_{2}, 0\right)$ on $\Gamma$. By Proposition 2.6 there exists a sequence $\psi^{j} \in C_{0}^{\infty}\left(\omega ; \mathbb{R}^{3}\right)$ such that, for $v^{j}:=y+\psi^{j}$, the sequence $D_{\alpha} v^{j}$ generates $v, v^{j} \rightarrow y$ weakly in $W^{1, p}\left(\omega ; \mathbb{R}^{3}\right)$ and the sequence $\left\{\left|D_{\alpha} v^{j}\right|^{p}\right\}_{j}$ is equi-integrable. By a measurable selection argument (see for instance Buttazzo [14, Proposition 2.2.7]), there exists a sequence of measurable functions $z^{j}: \omega \rightarrow \mathbb{R}^{3}$ such that

$$
\begin{aligned}
W_{0}\left(D_{\alpha} v^{j}\left(x_{\alpha}\right)\right) & =\min \left\{W\left(D_{\alpha} v^{j}\left(x_{\alpha}\right), z\right): z \in \mathbb{R}^{3}\right\} \\
& =W\left(D_{\alpha} v^{j}\left(x_{\alpha}\right) \mid z^{j}\left(x_{\alpha}\right)\right) \quad \text { a.e. } x_{\alpha} \in \omega .
\end{aligned}
$$

By the upper bound on $W$ we have

$$
-c \leqslant W_{0}\left(D_{\alpha} v^{j}\right)=W\left(D_{\alpha} v^{j} \mid z^{j}\right) \leqslant W\left(D_{\alpha} v^{j} \mid 0\right) \leqslant C\left(\left|D_{\alpha} v^{j}\right|^{p}+1\right)
$$

almost everywhere, and therefore the sequence $W_{0}\left(D_{\alpha} v^{j}\right)$ turns out to be equi-integrable. By continuity of $W_{0}$ and an application of Theorem 2.2 we get

$$
\begin{aligned}
\lim _{j \rightarrow \infty} \int_{\Omega} W\left(D_{\alpha} v^{j} \mid z^{j}\right) \mathrm{d} x & =\lim _{j \rightarrow \infty} \int_{\omega} W_{0}\left(D_{\alpha} v^{j}\left(x_{\alpha}\right)\right) \mathrm{d} x_{\alpha} \\
& =\int_{\omega} \int_{M^{3 \times 2}} W_{0}(\bar{F}) \mathrm{d} v_{x_{\alpha}}(\bar{F}) \mathrm{d} x_{\alpha}
\end{aligned}
$$

Let us remark moreover that, by the lower bound on $W$, even the sequence $z^{j}$ turns out to be $p$-equi-integrable.

Let $\bar{w}^{j}$ be functions in $C_{0}^{\infty}\left(\omega ; \mathbb{R}^{3}\right)$ such that

$$
\left\|z^{j}-(0,0,1)-\bar{w}^{j}\right\|_{p}<1 / j
$$

and let $\varepsilon_{n_{j}}$ be a subsequence of $\varepsilon_{n}$ such that

$$
\left\|\varepsilon_{n_{j}} D_{\alpha} \bar{w}^{j}\right\|_{p} \rightarrow 0
$$

We define $w^{j}:=(0,0,1)+\bar{w}^{j}$. Then $w^{j}=(0,0,1)$ on $\Gamma, w^{j}$ is $p$-equi-integrable and

$$
\lim _{j \rightarrow \infty} \int_{\Omega}\left[W\left(D_{\alpha} v^{j} \mid z^{j}\right)-W\left(D_{\alpha} v^{j}+\varepsilon_{n_{j}} x_{3} D_{\alpha} w^{j} \mid w^{j}\right)\right] \mathrm{d} x=0 .
$$


Indeed, the sequences $\left(D_{\alpha} v^{j} \mid z^{j}\right)$ and $\left(D_{\alpha} v^{j}+\varepsilon_{n_{j}} x_{3} D_{\alpha} w^{j} \mid w^{j}\right)$ generate the same Young measure and, being $p$-equi-integrable, the upper bound on $W$ ensures that the sequences $W\left(D_{\alpha} v^{j} \mid z^{j}\right)$ and $W\left(D_{\alpha} v^{j}+\varepsilon_{n_{j}} x_{3} D_{\alpha} w^{j} \mid w^{j}\right)$ are equi-integrable as well. Thus 8.3 follows by an application of Theorem 2.2. Let us define $y^{j}$ by

$$
y^{j}(x):=v^{j}\left(x_{\alpha}\right)+\varepsilon_{n_{j}} x_{3} w^{j}\left(x_{\alpha}\right)
$$

and observe that, using 8.3 and 8.2 , we have

$$
\begin{aligned}
\lim _{j \rightarrow \infty} \int_{\Omega} W\left(D_{\alpha} y^{j} \mid \frac{D_{3} y^{j}}{\varepsilon_{n_{j}}}\right) \mathrm{d} x & =\lim _{j \rightarrow \infty} \int_{\Omega} W\left(D_{\alpha} v^{j}+\varepsilon_{n_{j}} x_{3} D_{\alpha} w^{j} \mid w^{j}\right) \mathrm{d} x \\
& =\lim _{j \rightarrow \infty} \int_{\Omega} W\left(D_{\alpha} v^{j} \mid z^{j}\right) \mathrm{d} x=\int_{\omega} \int_{M^{3 \times 2}} W_{0}(\lambda) \mathrm{d} v_{x_{\alpha}}(\lambda) \mathrm{d} x_{\alpha} .
\end{aligned}
$$

Moreover, since $y^{j} \rightarrow \tilde{y}$ in $L^{p}\left(\Omega ; \mathbb{R}^{3}\right)$, where $\tilde{y}\left(x_{1}, x_{2}, x_{3}\right)=y\left(x_{1}, x_{2}\right)$, we also deduce that

$$
\lim _{j \rightarrow \infty} \int_{\Omega} f(x) \cdot y^{j}(x) \mathrm{d} x=\int_{\Omega} f(x) \cdot \tilde{y}\left(x_{\alpha}\right) \mathrm{d} x=\int_{\omega} \int_{-1 / 2}^{1 / 2} f\left(x_{\alpha}, x_{3}\right) \mathrm{d} x_{3} \cdot y\left(x_{\alpha}\right) \mathrm{d} x_{\alpha} .
$$

Thus, the first part of the proof is concluded by noting that

- $y^{j}=\left(x_{1}, x_{2}, \varepsilon_{n_{j}} x_{3}\right)$ on $\Gamma$,

- $\mu^{j}:=\delta_{D y^{j}} \rightarrow v \otimes \delta_{0}$ weak $^{*}$ in $L_{w}^{\infty}\left(\Omega ; \mathcal{M}\left(M^{3 \times 3}\right)\right)$,

- $q\left(\mu^{j}\right) \rightarrow q\left(v \otimes \delta_{0}\right)=v$ weak $^{*}$ in $L_{w}^{\infty}\left(\omega ; \mathcal{M}\left(M^{3 \times 2}\right)\right)$ by continuity of $q$,

and using Remark 8.3

The next step consists in proving the liminf inequality for the sequence $I_{\mathcal{E}_{n}}^{\mathcal{M}}$. Let $\mu^{n} \in$ $L_{w}^{\infty}\left(\Omega ; \mathcal{M}\left(M^{3 \times 3}\right)\right)$ be a sequence such that $q\left(\mu^{n}\right)$ converges weak* to $v$ in $L_{w}^{\infty}\left(\omega ; \mathcal{M}\left(M^{3 \times 2}\right)\right)$. We have to prove that

$$
\liminf _{n \rightarrow \infty} I_{\varepsilon_{n}}^{\mathcal{M}}\left(\mu^{n}\right) \geqslant I(v) .
$$

Without loss of generality we may suppose that the left hand side of the inequality above is finite and that the liminf is indeed a limit. Then $\sup _{n} I_{\varepsilon_{n}}^{\mathcal{M}}\left(\mu^{n}\right)<+\infty$ and, by Lemma 5.1 there exist $\mu \in \dot{\mathcal{Y}}_{\Gamma}^{1, p}\left(\Omega ; \mathbb{R}^{3}\right)$ and a subsequence $\mu^{n_{k}}$ such that

$$
\mu^{n_{k}} \rightarrow \mu \quad \text { weak }^{*} \text { in } L_{w}^{\infty}\left(\Omega ; \mathcal{M}\left(M^{3 \times 3}\right)\right)
$$

and the corresponding underlying deformations $y^{n_{k}}$ and $y$ which satisfy the boundary conditions $y^{n_{k}}(x)=\left(x_{1}, x_{2}, \varepsilon_{n_{k}} x_{3}\right)$ and $y(x)=\left(x_{1}, x_{2}, 0\right)$ are such that

$$
y^{n_{k}} \rightarrow y \quad \text { weakly in } W^{1, p}\left(\Omega ; \mathbb{R}^{3}\right), \quad y=y\left(x_{1}, x_{2}\right) .
$$

Hence, by Remark $8.3 v \in q\left(\dot{\mathcal{Y}}_{\Gamma}^{1, p}\left(\Omega ; \mathbb{R}^{3}\right)\right)=\mathcal{Y}^{1, p}\left(\omega ; \mathbb{R}^{3}\right)$. Moreover,

$$
\begin{aligned}
\liminf _{n \rightarrow \infty} I_{\varepsilon_{n}}^{\mathcal{M}}\left(\mu^{n}\right) & =\lim _{n \rightarrow \infty} I_{\varepsilon_{n}}^{\mathcal{M}}\left(\mu^{n}\right)=\lim _{k \rightarrow \infty} I_{\varepsilon_{n_{k}}}^{\mathcal{M}}\left(\mu^{n_{k}}\right) \\
& =\lim _{k \rightarrow \infty}\left(\int_{\Omega} W\left(D_{\alpha} y^{n_{k}} \mid \frac{D_{3} y^{n_{k}}}{\varepsilon_{n_{k}}}\right) \mathrm{d} x-\int_{\Omega} f \cdot y^{n_{k}} \mathrm{~d} x\right)
\end{aligned}
$$




$$
\begin{aligned}
& \geqslant \lim _{k \rightarrow \infty}\left(\int_{\Omega} W_{0}\left(D_{\alpha} y^{n_{k}}\right) \mathrm{d} x-\int_{\Omega} f \cdot y^{n_{k}} \mathrm{~d} x\right) \\
& =\lim _{k \rightarrow \infty}\left(\int_{\Omega}\left\langle\bar{\pi}_{\#} \mu_{x}^{n_{k}}, W_{0}\right\rangle \mathrm{d} x-\int_{\Omega} f(x) \cdot y^{n_{k}}(x) \mathrm{d} x\right) \\
& =\lim _{k \rightarrow \infty}\left(\int_{\omega}\left(\int_{-1 / 2}^{1 / 2}\left\langle\bar{\pi}_{\#} \mu_{x}^{n_{k}}, W_{0}\right\rangle \mathrm{d} x_{3}\right) \mathrm{d} x_{\alpha}-\int_{\Omega} f(x) \cdot y^{n_{k}} \mathrm{~d} x\right) \\
& =\lim _{k \rightarrow \infty}\left(\int_{\omega}\left\langle\mathrm{Av}^{3} \bar{\pi}_{\#} \mu^{n_{k}}, W_{0}\right\rangle \mathrm{d} x_{\alpha}-\int_{\Omega} f(x) \cdot y^{n_{k}}(x) \mathrm{d} x\right) \\
& =\lim _{k \rightarrow \infty}\left(\int_{\omega}\left\langle q\left(\mu^{n_{k}}\right)_{x_{\alpha}}, W_{0}\right\rangle \mathrm{d} x_{\alpha}-\int_{\Omega} f(x) \cdot y^{n_{k}}(x) \mathrm{d} x\right) \\
& \geqslant \int_{\omega}\left\langle v_{x_{\alpha}}, W_{0}\right\rangle \mathrm{d} x_{\alpha}-\int_{\omega} \int_{-1 / 2}^{1 / 2} f\left(x_{\alpha}, x_{3}\right) \mathrm{d} x_{3} \cdot y\left(x_{\alpha}\right) \mathrm{d} x_{\alpha},
\end{aligned}
$$

where in the last inequality we have used 8.4 and Theorem 2.2

\section{REFERENCES}

1. Acerbi, E., Buttazzo, G., \& Percivale, D. A variational definition of the strain energy for an elastic string. J. Elasticity 25 (1991), 137-148. Zbl 0734.73094 MR 1111364

2. ACERBi, E. \& FUSCO, N. Semicontinuity problems in the calculus of variations. Arch. Rat. Mech. Anal. 86 (1984), 125-145. Zbl 0565.49010 MR 0751305

3. Anzellotti, G., Baldo, S., \& Percivale, D. Dimension reduction in variational problems, asymptotic development in $\Gamma$-convergence and thin structures in elasticity. Asymptot. Anal. 9 (1994), 61-100. Zbl 0811.49020 MR 1285017

4. BALL, J. M. A version of the fundamental theorem for Young measures. PDEs and Continuum Models of Phase Transitions, Lecture Notes in Phys. 344, Springer (1989), 207-215. Zbl 0991.49500 MR 1036070

5. BALl, J. M. \& JAMES, R. D. Fine phase mixtures as minimizers of energy. Arch. Rat. Mech. Anal. 100 (1987), 13-52. Zbl 0629.49020 MR 0906132

6. BALL, J. M. \& JAMES, R. D. Proposed experimental tests of a theory of fine microstructure and the two well problem. R. Soc. London Philos. Trans. Ser. A 338 (1992), 389-450. Zbl 0758.73009

7. BĚLíK, P. \& LUSKIN, M. A computational model for the indentation and phase transformation of a martensitic thin film. J. Mech. Phys. Solids 50 (2002), 1789-1815. Zbl 1004.74059 MR 1915331

8. BĚLíK, P. \& LUSKIN, M. A total-variation surface energy model for thin films of martensitic cristals. Interfaces Free Bound. 4 (2002), 71-88. Zbl 1014.49013 MR 1877536

9. Bhattacharya, K. \& Braides, A. Thin films with many small cracks. R. Soc. London Proc. Ser. A Math. Phys. Eng. Sci. 458 (2002), 823-840. Zbl 1011.74042 MR 1898090

10. Bhattacharya, K. \& James, R. D. A theory of thin films of martensitic materials with applications to microactuators. J. Mech. Phys. Solids 47 (1999), 531-576. Zbl 0960.74046 MR 1675215

11. Bocea, M. Young measures minimizers in the asymptotic analysis of thin films. Preprint (2003).

12. BocEA, M. \& FONSECA, I. Equi-integrability results for 3D-2D dimension reduction problems. ESAIM Control Optim. Calc. Var. 7 (2002), 443-470. Zbl pre01966585 MR 1925037

13. Braides, A., Fonseca, I., \& Francfort, G. 3d-2d asymptotic analysis for inhomogeneous thin films. Indiana Univ. Math. J. 49 (2000), 1367-1404. Zbl 0987.35020 MR 1836533

14. Buttazzo, G. Semicontinuity, Relaxation and Integral Representation in the Calculus of Variations. \begin{tabular}{l|l|l|} 
Pitman Res. Notes Math. Ser. 207, Longman (1989). Zbl 0669.49005 & MR 1020296 \\
\hline
\end{tabular} 
15. Ciarlet, P. G. \& Destuynder, P. A justification of the two-dimensional linear plate model. J. Mécanique 18 (1979), 315-344. Z Zbl 0415.73072 MR 0533827

16. Dacorogna, B. Direct Methods in the Calculus of Variations. Appl. Math. Sci. 78, Springer (1989). Zbl 0703.49001 MR 0990890

17. Dal Maso, G. An Introduction to $\Gamma$-convergence. Birkhäuser (1993). Zbl 0816.49001 MR 1201152

18. Freddi, L. \& PARONI, R. A 3D-1D Young measure theory of an elastic string. Asymptot. Anal. 39 (2004), 61-89. MR 2083576

19. James, R. D. \& Kinderlehrer, D. Theory of diffusionless phase transitions. PDEs and Continuum Models of Phase Transitions, Lecture Notes in Phys. 344, Springer (1989), 51-84. Zbl 0991.74504 MR 1036063

20. Kinderlehrer, D. \& Pedregal, P. Characterizations of Young measures generated by gradients. Arch. Rat. Mech. Anal. 115 (1991), 329-365. Zbl 0754.49020 MR 1120852

21. Kinderlehrer, D. \& Pedregal, P. Gradient Young measures generated by sequences in Sobolev spaces. J. Geom. Anal. 4 (1994), 59-90. Zbl 0808.46046 MR 1274138

22. Le Dret, H. \& Raoult, A. The nonlinear membrane model as variational limit of nonlinear threedimensional elasticity. J. Math. Pures Appl. 74 (1995), 549-578. Zbl 0847.73025 MR 1365259

23. Luskin, M. On the computation of crystalline microstructure. Acta Numer. 5 (1996), 191-257. Zbl 0867.65033 MR 1624603

24. MÜLlER, S. Variational models for microstructure and phase transitions. Calculus of Variations and Geometric Evolution Problems (Cetraro, 1996), Lecture Notes in Math. 1713, Springer (1999), 85-210. Zbl 0968.74050 MR 1731640

25. Murat, F. \& TARTAR, L. Calculus of variations and homogenization. Topics in the Mathematical Modelling of Composite Materials, Progr. Nonlinear Differential Equations Appl. 31, Birkhäuser (1997), 139173. Zbl 0939.35019 MR 1493042

26. Paroni, R. From discrete to continuum problems: a Young measure approach. Z. Angew. Math. Phys. 54 (2003), 328-348. Zbl pre01968509 MR 1967332

27. Pedregal, P. Parametrized Measures and Variational Principles. Progr. Nonlinear Differential Equations Appl. 130, Birkhäuser (1997). Zbl 0879.49017 MR 1452107

28. Santos, P. M. \& Zappale, E. Second-order analysis for thin structures. Nonlinear Anal. 56 (2004), 679-713. Zbl pre02056770 MR 2036786

29. SHu, Y. C. Heterogeneous thin films of martensitic materials. Arch. Rat. Mech. Anal. 153 (2000), 39-90. Zbl 0959.74043 MR 1772534

30. VAlADIER, M. Young measures. Methods of Nonconvex Analysis, Lecture Notes in Math. 1446, Springer (1990), 152-188. Zbl 0738.28004 MR 1079763

31. Young, L. C. Lectures on the Calculus of Variations and Optimal Control Theory. W. B. Saunders (1969). Zbl 0177.37801 MR 0259704 